\title{
The Effects of Combined Exercises Intensity (Aerobics- Resistance) on Plasma Cortisol and Testosterone Levels in Active Males
}

\author{
Gholamali Ali Sholi', Mohsen Ghanbarzadeh ${ }^{2 *}$, Abdolhamid Habibi ${ }^{3}$, Rohellah Ranjbar ${ }^{2}$ \\ ${ }^{1}$ MSc, Department of Exercise and Sport Physiology, Faculty of Sport Science, Shahid Chamran University of Ahwaz, Ahwaz, \\ Iran \\ ${ }^{2}$ Assistant Professor, Department of Exercise and Sport Physiology, Faculty of Sport Science, Shahid Chamran University of \\ Ahwaz, Ahwaz, Iran \\ ${ }^{3}$ Associate Professor, Department of Exercise and Sport Physiology, Faculty of Sport Science, Shahid Chamran University of \\ Ahwaz, Ahwaz, Iran
}

\section{*Correspondence to \\ Mohsen Ghanbarzadeh; Assistant \\ Professor at Department of Exercise and Sport Physiology, Faculty of Sport Science, Shahid Chamran Uni- versity of Ahwaz, Ahwaz, Iran. Tell: +989163096612 Fax: +986133336316 Email: \\ ghanbarzadeh213@gmail.com}

Published online 29 June 2016

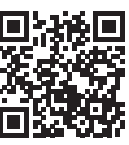

Please cite this article as follows: Ali Sholi G, Ghanbarzadeh M, Habibi A, Ranjbar R. The effects of combined exercises intensity (aerobics-resistance) on plasma cortisol and testosterone levels in active males. Int J Basic Sci Med. 2016;1(1):1824. doi:10.15171/ ijbms.2016.05

\begin{abstract}
Background: Changes in hormone levels especially testosterone and cortisol occur as a result of exercise intensity. In other words, the exercise intensity significantly influences these changes. The aim of this study was to investigate the effects of aerobics-resistance (concurrent) exercise intensities on the serum testosterone and cortisol levels in the active men.

Methods: In this clinical trial, 10 healthy men were included in their combined activities in low, medium, and high levels. Different intensities (aerobic activity such as running on a treadmill at a speed of $8,6.9$, and $11.2 \mathrm{mph}$ and resistance exercise on $45 \%, 65 \%$, and $85 \%$ of maximum strange, maximum of six moves) were considered for all participants for unity. Blood samples were measured in three phases (baseline, immediately after exercise, and 24 hours after training contracts) at three levels. Two-way analysis of variance (ANOVA) with repeated normal data and Bonferroni post hoc test were conducted.

Results: The results showed that cortisol and plasma lactate concentration was declined immediately before and 24 hours after the combined exercise with the significant level of activity $(P<0.05)$. Joint action at different intensities resulted in significant changes not in testosterone level $(P<0.05)$.

Conclusion: In this study, the intensity of training as an independent variable influencing the changes in the levels of testosterone and cortisol has been recommended; so highly sportaffected hormonal changes during the combined exercise should be considered more than that in one exercise.

Keywords: Concurrent exercise, Testosterone, Cortisol, Plasma lactate, Exercise intensities
\end{abstract}

\section{Introduction}

One of the most important methods for determining the exercise intensity on the athletes to measure biochemical variables is measurement of enzymes, hormones, and metabolites. ${ }^{1}$ In line with the theme of this study, Agha-Alinejad et al showed that endurance training exercise can increase the secretion of cortisol in the blood. ${ }^{2}$ Shakeri et al found that total testosterone secretion depends on the volume and intensity of exercise. ${ }^{3}$ Beni et al after eight weeks of aerobic exercise measured the levels of testosterone and cortisol in young men and showed that after eight weeks of aerobic training, testosterone levels sig- nificantly increased while cortisol levels decreased. ${ }^{4}$ Enhancing physical activity as a potent stimulation of known endocrine system depends on hormonal sensitivities to exercise intensity, and duration and type of exercise. ${ }^{5}$ The most important regulator of testosterone and cortisol are consistent practice. ${ }^{6}$ Ratios of testosterone to cortisol express the balance of anabolic and catabolic muscle metabolisms. ${ }^{7}$ The anabolic hormone (testosterone) along with protein synthesis and muscle strength increase bone density and stimulate strength. ${ }^{8,9}$ Enhancing the interaction between the cortisol and testosterone as a neutral physical activity is known. At the beginning of

Copyright (C) 2016 The Author(s); Published by Zabol University of Medical Sciences. This is an open-access article distributed under the terms of the Creative Commons Attribution License (http://creativecommons.org/licenses/by/4.0), which permits unrestricted use, distribution, and reproduction in any medium, provided the original work is properly cited. 
long-term exercise training, testosterone increases and with continued operation, it decreases. The endocrine compliance regulates the muscle activity, stimulates the glycogenolysis, and facilitates the gluconeogenesis. ${ }^{10}$ Glucocorticoid cortisol produced by the adrenal glands is the main, most important anti-stress hormone in the body. However, the increase in long-term problems causes immune system problems and analysis of their most important proteins. ${ }^{11}$ The cortisol present in the muscle and fat tissue that breaks down protein into amino acids and glycerol is a catabolic hormone that affects free fatty acids in the body by hydrolysis of triglyceride.

Therefore, cortisol can be a powerful hormone to limit skeletal muscle development. ${ }^{12,13}$ Instead of an increase in testosterone level, muscle hypertrophy and increased strength can also be a result of a drop in cortisol level. In either case, maintaining and increasing the concentration of testosterone in circulation along with a drop in cortisol level could be a major factor in promoting muscle hypertrophy resistance training program followed by chronic or severe exercise. ${ }^{14}$ Both resistance training and aerobic training programs are interesting and useful for competitive and recreational athletes. Doing a lot of training programs at the same time catches that in both forms of normal exercise. ${ }^{15}$ Aerobic exercise causes some physiological adaptations such as maximal aerobic power and efficiency of the cardiovascular system. ${ }^{16}$ Resistance training increases muscle mass and muscle strength. ${ }^{17}$ Practicing with several energy devices and multi tasking types of exercise (resistance training and aerobics) are called parallel or combination exercises. A combination of separate aerobic and resistance training exercises improve body composition and cardiovascular health factors $^{18}$ and develop physical fitness and metabolic health. ${ }^{19}$ Concurrent strength and endurance training, compared with both style training activities alone, leads to the disruption of compatibility. This feature is known as the effect of concurrent training or interaction. ${ }^{20}$ Powerful stimulant acute resistance exercise increases the concentration of testosterone and cortisol. However, the combined activities could have higher metabolic demand and thus lead to a further increase in cortisol, which can negatively affect the release of testosterone during a training session. ${ }^{21}$ It seems that testosterone and cortisol respond to higher intensity and longer duration of activity. ${ }^{22,23}$ High-intensity training protocols involving large muscles acutely increase testosterone level, ${ }^{24}$ and also when the exercise intensity decreases from $70 \%$ to $40 \%$, hormonal responses decrease in both sexes. ${ }^{25}$ Testosterone secretion of glucocorticoids depends on especially cortisol synthesis. ${ }^{26}$ Changes in serum cortisol are dependent on the type, intensity, and duration of activity so that one of the most significant drivers of secretion of hormones is severe physical activity. Intense physical activity increases the secretion of adrenocorticotropic hormone (ACTH), and cortisol secretion is increased as a result. However, if the activity is below the threshold intensity, cortisol levels may not increase above resting levels and may decrease. ${ }^{27}$ Rosa et al in their study of the effect of compound activity on serum cortisol in healthy men concluded that both activities regardless of their sequence which reduced the cortisol level depend on the intensity of exercise. ${ }^{28}$ In contrast, Cruz et al in their study on obese adults exam- ined cortisol after combination exercise. Use of adequate combination intensity exercise does not increase cortisol level in young obese adults. ${ }^{29}$ Cadore et $\mathrm{al}^{30}$ examined the physiological effects on testosterone and cortisol activity in combination with different sequences. Testosterone level significantly increased immediately after aerobic activities and resistance exercises. Cortisol returned to resting level immediately after exercise in both groups. The inverse relationship between cortisol and testosterone can be observed. Therefore, the lower levels of cortisol increase testosterone levels. Thus, physical activity is effective in increasing testosterone. It is assumed that if total energy expenditure (in step aerobics activities) and workload (at the stage of resistance exercise) combine during an exercise at different intensities, the response is possibly similar to these hormones. This study aimed to investigate the effect of combined aerobic-resistance exercises on the amount of active testosterone and cortisol in men.

\section{Methods}

Subjects included 10 male physical education students of Shahid Chamran University of Ahwaz. Subject selection was based on a homogeneous condition. Fifty-six students attended as the first applicants, and then 14 subjects were accepted with homogeneous characteristics of anthropometric and body composition, weight, and height. The subjects were selected voluntarily and they agreed to the terms written in this clinical trial. Following the implementation of the project, 4 out of 14 were eliminated due to lack of continuous operation in the implementation of clinical sports trials. Finally, the study periods of the test data of 10 individuals eligible to participate in the project were measured and recorded from the beginning to end. Having active regular exercise (more than one session per week) in the last 6 months and the subjects' medical records showed that subjects did not have any history of cardiovascular disease or dysfunction of the immune system. In addition, participants with infectious diseases or taking any medication associated with inflammation during the study were excluded from the study, along with participants who were absent in the exercise or had flu. The participants were asked if they used performance-enhancing drugs and other medicines that could affect the research. The participants also were given the necessary information about the purpose and risks of the study. Subjects who met the inclusion criteria provided informed consent. In four sessions, the participants went to the laboratory of Physical Education. In the first test of maximum oxygen consumption (VO2max), maximum power or one repetition maximum (1RM), body composition, and anthropometric dimensions were measured. During joint action before the sessions (sessions II, III, and IV), the subjects were asked to refrain from any strenuous activity for 72 hours. In addition, participants were asked to report any disease before completing the report. Accordingly, acute fluctuations of inflammatory markers were minimized before collecting venous blood samples. After at least 10 hours of overnight fast, subjects were referred at 7:30 AM-7:45 AM to the laboratory for acute combined activities. After taking the initial blood sample of subjects, intensities of activity in the form of a cross combination within 7 days and at the end of each session, at the end of 24-hour blood samples, were measured. The 
concentrations of hormones were measured in comparison to baseline (prior to exercise) for different groups of subjects, heavily used in combination activities, including low, medium, and high intensities. Joint action at different intensities on the aerobic energy costs and workload on stage matched the resistance. All sessions combined activities for each subject at the same time and at a temperature of $20-25^{\circ} \mathrm{C}$ and relative humidity of $30 \%$ to $35 \%$ in the laboratory of Physical Education. Research outline is shown in Table 1.

Anthropometric characteristics, body composition, height, and weight of the subjects were measured with an accuracy of $5 \mathrm{~mm}$ and $2.0 \mathrm{~kg}$. Body mass index (BMI) was calculated by dividing weight by squared height $\left(\mathrm{kg} / \mathrm{m}^{2}\right)$. Body composition was measured using a spectrophotometer (Olympia version 3.3, Guan Company, South Korea). Maximum power was measured via one repetition maximum (1RM) in six bench press, leg press, barbell biceps, leg curl, lat pull down machine, and the front of the hip using Berziki formula. ${ }^{31}$ [(Number of repetitions) 0278 / 0-0278/1] / Weight shifted 1RM = maximal oxygen uptake (VO2max). To determine VO2max, subjects' Bruse protocol was performed on the treadmill (model Saturn, $\mathrm{h} / \mathrm{p} /$ cosmos, Germany) and related data were collected through exchange respiratory gases continuously using a gas analyzer (model Gunshorn, Germany) and LS8 software; the $4.10 \mathrm{mph}$ arrives. For measuring testosterone and cortisol levels, blood samples were collected from the brachial vein $(5 \mathrm{~mL})$ following overnight fast (before exercise), immediately after and 24 hours after exercise activity in the second, third, and fourth sessions. Plasma samples were stored at $70^{\circ} \mathrm{C}$ until use for measuring testosterone and cortisol levels. Testosterone and cortisol were measured (using a commercial kit 96 test-ELISA-human-testosterone-D.plas, 96-test kits, ELISA-human-cortisol -D.plas) with ELISA reading device Biotech ELX800 models (USA). ${ }^{15}$ After a brief warm-up, subjects combined activities while the aerobic stage began before the resistance stage. Aerobic activity step and energy intake of $300 \mathrm{kcal}$ for each compound were per individual. ${ }^{32}$ In order to compare different intensities of activity combined with aerobic phase, the subjects ran on a treadmill at speeds of $5 \mathrm{mph}(8 \mathrm{kph})$ for low intensity, $6 \mathrm{mph}(6.9$ $\mathrm{kph})$ for moderate, and $7 \mathrm{mph}(11.2 \mathrm{kph})$ for high intensity. ${ }^{33}$ Duration of aerobic stage was separately calculated for each individual based on the following formula: $\mathrm{kcal}=$ MET $\times$ weight $(\mathrm{kg}) \times$ time $(\mathrm{h}){ }^{34}$ Matt (metabolic equivalent of task) values used for low intensity, medium, and high aerobic stage were 8, 10, and 5.11, respectively. ${ }^{33}$ For resistance exercise, six subjects did the front thigh in the bench press, leg press, barbell biceps, leg curl, lat pull down machine. To measure the capacity of resistance activity of each participant in the study, the following criteria were used: the total volume $(\mathrm{kg})=$ the number of sets of repetitions $\times$ amount of weight $(\mathrm{kg}){ }^{35}$ The weight-intensity resistance values at low, medium, and high had one repetition maximum $45 \%, 65 \%$, and $85 \%$, respectively. Each movement was repeated three times, and the number of repetitions per set for each move was obtained by the above formula, ${ }^{35}$ between low, medium, and high sets 1,2, and 3 minutes between the three minutes movements. Descriptive statistics were used to calculate the mean and standard deviation of variables. In addition, to evaluate the frequency, the Shapiro-Wilk test was used to test research hypotheses via two-way analysis of variance (ANOVA) with repeated measures and post hoc Bonferroni. And to study parameters with each other at extremely low, moderate, and high intensities, Pearson correlation test was used. All statistical analyses were done using SPSS version 17.0 when the level of 0.05 was considered significant.

\section{Results}

Subjects' features were as follows: mean age $(15.1 \pm 3.20$ years), height $(7.5 \pm 172 \mathrm{~cm})$, weight $(64.5 \pm 28.64 \mathrm{~kg})$, and BMI (73.1 \pm 88.21$)$. Anthropometric indices, body composition, and physiological features of subjects are shown in Table 2.

Data on glucose, lactate and total plasma protein before, immediately after, and 24 hours after a meeting of different intensities of joint action are shown in Table 3.

Analysis of the results showed that the lactate concentrations at different times in each sampling and at different intensities were significantly different $(P=0.001)$. Bonferroni post hoc test showed a significant difference between lactate concentration in the subjects immediately after the activity and 24 hours after activities per se highly active combination $(P<0.05)$. Analysis of the results also showed that the subjects' glucose and plasma total protein did not significantly change immediately after exercise and 24 hours after exercise compared to before combination exercise in different intensities of the activities $(P<0.05)$. A significant difference was seen between different intensities of exercise combined with active exercise immediately after and 24 hours after regarding the concentration of lactate, glucose, and total plasma protein of subjects $(P<0.05)$. Combined testosterone response to an acute exercise session with different intensities is presented in Figure 1. The findings showed that testosterone levels immediately after the exercise of participants and 24 hours after exercise compared with before combination exercise did not significantly change in different intensities of activities $(P<0.05)$. A significant difference was observed in the testosterone concentration of subjects regarding different intensities of combination exercises immediately after and 24 hours after exercise $(P<0.05$; Figure 1$)$.

Joint action did not show significant differences in testosterone concentrations with respect to the intensity of meeting immediately after, and 24 hours after the exercise of the participants $(P>0.05)$.

Table 1. Schematic Representation of Research

\begin{tabular}{|c|c|c|c|c|}
\hline \multirow{3}{*}{ Start } & $\begin{array}{l}\text { Blood samples before } \\
\text { exercise }\end{array}$ & Low-intensity activity & Blood samples immediately after exercise & $\begin{array}{l}\text { Blood samples } 24 \text { hours after } \\
\text { exercise }\end{array}$ \\
\hline & $\begin{array}{l}\text { Blood samples before } \\
\text { exercise }\end{array}$ & Middle-intensity activity & Blood samples immediately after exercise & $\begin{array}{l}\text { Blood samples } 24 \text { hours after } \\
\text { exercise }\end{array}$ \\
\hline & $\begin{array}{l}\text { Blood samples before } \\
\text { exercise }\end{array}$ & High-intensity activity & Blood samples immediately after exercise & $\begin{array}{l}\text { Blood samples } 24 \text { hours after } \\
\text { exercise }\end{array}$ \\
\hline
\end{tabular}


Table 2. Mean and SD of Anthropometric Characteristics, Body Composition, and Physiological Features of Active Men

\begin{tabular}{lc}
\hline Variables & Mean \pm SD \\
\hline Age $($ years $)$ & $20.30 \pm 1.15$ \\
Height $(\mathrm{cm})$ & $172 \pm 5.70$ \\
Weight $(\mathrm{kg})$ & $64.28 \pm 5.64$ \\
$\mathrm{BMI}\left(\mathrm{kg} / \mathrm{m}^{2}\right)$ & $21.88 \pm 1.73$ \\
Maximum oxygen consumption $(\mathrm{ml} / \mathrm{kg} / \mathrm{min})$ & $48.93 \pm 3.03$ \\
Fat percentage $(\%)$ & $15.25 \pm 3.61$ \\
\hline
\end{tabular}

Abbreviations: BMI, Body mass index; SD, standard deviation.

Cortisol response to acute exercise in combination with different intensities is presented in Figure 2. The findings showed that the subjects' cortisol levels did not significantly differ immediately before and 24 hours after exercise compared to activity at different intensities of joint action $(P<0.05)$. The results showed that the combination of different-intensity exercises was significantly different immediately after the exercise in cortisol levels of subjects $(P<0.05)$. However, 24 hours after the exercise, there was no significant difference in cortisol levels $(P<0.05)$. Bonferroni post hoc test revealed that cortisol levels were lower immediately after the exercise compared with before and 24 hours after exercise. As a result, post hoc test showed a drastic reduction regarding the intensity than the other two $(P<0.05$; Figure 2$)$.

As a result, post hoc test was shown to significantly reduce the intensity than the other two $(P>0.05)$.

\section{Discussion}

Decrease in anabolic hormones (testosterone) to catabolic ones (cortisol) plays an important role in reduction of physical strength, muscle mass, and aerobic power. ${ }^{36}$ The ratio of testosterone to cortisol response in exercise is also used to evaluate the performance of the predictive capacity; when the ratio is above, it represents some conditions; $30 \%$ or more reflects decrease in anabolic and catabolic conditions in the body. ${ }^{5,6,37}$ The findings of this study showed no significant difference in testosterone level immediately and 24 hours after different intensities of combination exercise. The study of Cadore et al included 30 minutes of aerobic activity on a bicycle ergometer at $75 \%$ of maximum heart rate and four resistance movements in three sets with eight reps at $75 \%$ of one repetition maximum (1RM), showing significant increase in testosterone during the first phase of blood taking (exercise) in both

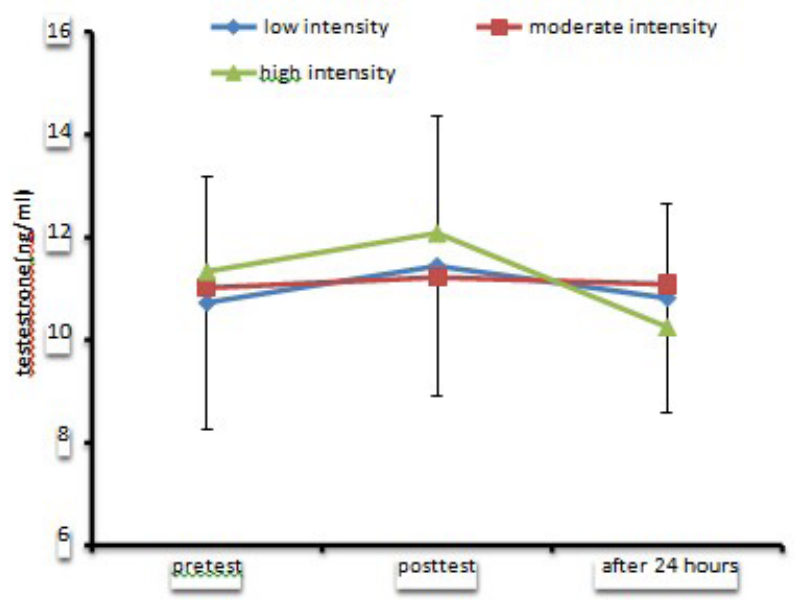

Figure 1. Mean Testosterone Levels in Different Intensities of Combination Exercises of Active Men.

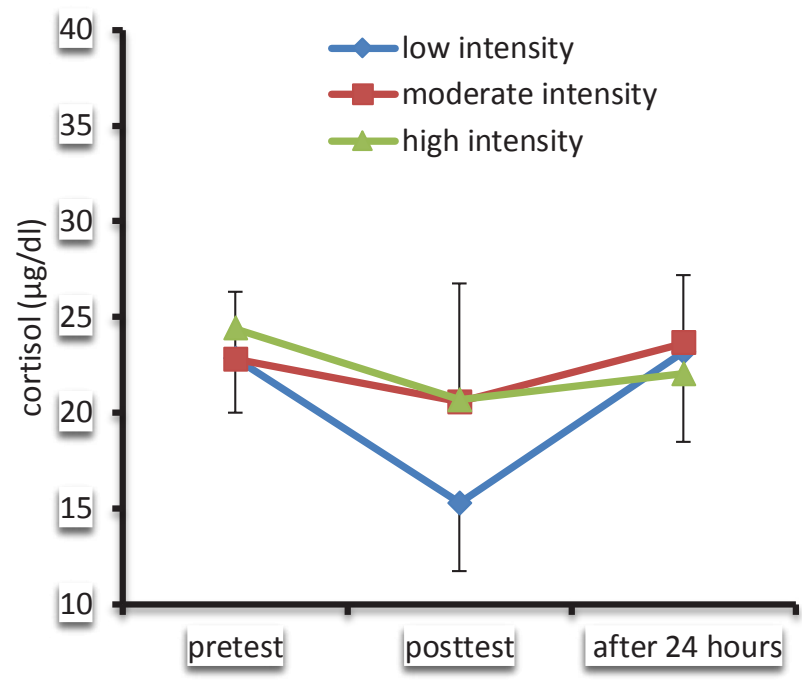

Figure 2. Average Cortisol Levels in Different Intensities of Combined Exercise.

aerobic-resistance exercise (AS) and resistance-aerobic exercise (SA), and in the second phase of blood taking, only immediately after exercise, a significant increase in AS group. During the meetings, AS and SA changes in testosterone levels after the first blood sample were larger than those in the second phase. ${ }^{30}$ In the study by Goto et

Table 3. Mean and SD of Lactic Acid, Glucose, and Plasma Total Protein Before, Immediately After, and 24 Hours After the Combined Activity (With Varying Intensities) Of Active Men

\begin{tabular}{lllll}
\hline & Variables & Before Training & Immediately After Training & Changing After 24 Hours \\
\hline \multirow{2}{*}{ Low-intensity } & Glucose $(\mathrm{mg} / \mathrm{dL})$ & $84.4 \pm 18.90$ & $78.1 \pm 17.30$ & $82.2 \pm 14.8$ \\
activity & Lactate $(\mathrm{mmol} / \mathrm{L})$ & $2.34 \pm 0.57$ & $6.91 \pm 1.58^{*}$ & $2.16 \pm 0.52 €$ \\
& Total protein $(\mathrm{mg} / \mathrm{dL})$ & $6.25 \pm 0.90$ & $6.14 \pm 0.28$ & $5.85 \pm 0.28$ \\
Middle-intensity & Glucose $(\mathrm{mg} / \mathrm{dL})$ & $15.70 \pm 82.3$ & $83.3 \pm 20.40$ & $90.5 \pm 14.60$ \\
activity & Lactate $(\mathrm{mmol} / \mathrm{L})$ & $0.58 \pm 2.13$ & $6.45 \pm 1.12^{*}$ & $1.98 \pm 0.95 €$ \\
\multirow{4}{*}{ High - intensity } & Total protein $(\mathrm{mg} / \mathrm{dL})$ & $5.99 \pm 0.35$ & $6.18 \pm 0.28$ & $5.84 \pm 0.54$ \\
activity & Glucose $(\mathrm{mg} / \mathrm{dL})$ & $87.20 \pm 16.50$ & $81.80 \pm 17.90$ & $89.90 \pm 15.50$ \\
& Lactate $(\mathrm{mmol} / \mathrm{L})$ & $2.09 \pm 0.33$ & $6.54 \pm 1.91^{*}$ & $192 \pm 0.35 €$ \\
\hline
\end{tabular}

Abbreviation: SD, standard deviation. 
al, no significant differences were observed in the concentrations of testosterone, cortisol, glucose, and lactate due to resistance exercise, aerobic activity with and without performance. ${ }^{38}$ In this study, changes were measured in testosterone levels in three different intensity levels (low $45 \%$, moderate $65 \%$, severe $85 \%$ ) equal to the amount of energy used per intensity immediately and 24 hours after exercise; none of these changes were significant. The Cadore et al results were inconsistent with the findings of Goto et al. ${ }^{38}$ Hormonal manipulation of various factors can increase testosterone level in response to optimized exercise, related to the impact of acute exercise session such as time and intensity, ${ }^{14,15,39}$ and interval ${ }^{16,40}$ hours of muscle mass. ${ }^{17,41}$ Long-term performance sets of numbers and compatibility with different training protocols may trigger hormonal responses to increase concentrations of hormones separately in order to optimize the compatibility with exercise protocols. ${ }^{19,20,39}$ The type of protocol used in this research can be stated because of inconsistent results, in addition to the synthesis of neurotransmitters' testosterone production (ARs) regarding the power of the synthesis of contractile proteins. ${ }^{14,22,23}$ Increased testosterone in response to resistance training appears to be related to the level of increase in the number of androgen receptors (ARs) in human cells. ${ }^{14}$ The content of ARs was not measured in this study, as it may change due to the interaction of circulating testosterone in the hormone receptors. Testosterone may result in an increase rather than a decrease in the secretion of the receptor binding. In fact, in practice, the number of ARs increased the resistance; also resistance exercise resulted in a sharp rise in the ARs content. ${ }^{14,26,27,42}$ The subjects in this study were physical education students, who were more interested in consistent aerobic exercise; therefore, one of the mentioned mechanism differences between the results can be attributed to this factor. However, this hypothesis must be considered with caution. The most likely hypothesis for the increase in resistance exercise stimulates the sympathetic nervous testosterone from the Leydig cells of the testes and adrenal glands and lactate..$^{31,32,42-45}$ Thus, no significant changes in the intensity of lactate after mixed activity may explain significant changes in testosterone concentrations. Approximately, 14\% decrease in plasma volume as one of the mechanisms of resistance exercise can increase testosterone concentrations. ${ }^{46}$ In fact, the increase in plasma levels would be wrong regarding changes resulting from the decline in testosterone concentrations, while the reduction in plasma volume may also reveal an increased circulating level of testosterone..$^{31,32,43,47}$ In this study, the change in total plasma protein was because of pooled activity at different intensities; joint action at different intensities resulted in no significant difference in total plasma protein as the main effect of intensity and timing of sampling. Thus, insignificant changes in the level of total plasma protein are consistent with no significant changes in testosterone. The findings showed that cortisol response after combination and interaction of different intensities of exercise (intensity $\times$ sampling) was not significantly different $(P<0.05)$. The results showed that cortisol levels significantly decreased immediately after exercise compared with before and 24 hours after the activity $(P<0.05)$. The findings regarding Cadore et al cortisol level are inconsistent with Cruz et al and Rosa et al. ${ }^{14,38,48,49}$ After low or high intensity, short-term or longterm exercises reduced when blood glucose levels and cortisol concentrations increased to maintain blood glucose level. ${ }^{13,49,50}$ Glucose levels were measured in the present study; the combined activities with different intensities did not lead to significant differences in glucose, possibly to justify significant changes in cortisol. By reducing the volume of plasma, cortisol concentration increases. ${ }^{13,49}$ Lack of significant changes in plasma total protein content showed no significant changes in cortisol level after different intensities of joint action in the study. Cortisol concentrations have shown similar responses among sedentary individuals, aerobic farmers, and resistance training conservatives. ${ }^{49-51}$ Insignificant changes in the cortisol level after exercise of the subjects were observed in active athletes with the above statement. The results can be different from sedentary subjects who had been justified with high BMI kg/m². ${ }^{25}$

Alghadir et al showed that increased levels of lactate at the end of practice changed cortisol levels during the rest of recovery. It is also possible that the duration of aerobic exercise is associated with changes in salivary and $\mathrm{LDH}^{52}$ As generally shown by Mohamadi et al and according to the results of present study, although the pattern of changing serum level of cortisol and testosterone hormones in both exercise groups is similar, in short-term protocols, LIBFR can increase catabolic-anabolic hormones greater than that of HIWBFR in young males. Hence, restricting blood flow during exercise is an important factor in making hormonal responses. However, because of the limited number of studies in this area, further studies are required for more precise understanding of the mechanisms involved in the hormonal responses to exercises. ${ }^{53}$ The level of cortisol in athletes was $1 \%$ higher during the resting. It should be noted that during exercise, the level of cortisol is associated with level of exercise intensity. The present study showed that one session of exhaustive exercise is not influential upon IL6 inflammatory marker and cortisol hormone. ${ }^{54}$

\section{Conclusion}

In general, the results showed that low intensity exercises combined with activities in men had no significant effect on testosterone levels; only the active males' cortisol levels had significant changes immediately after the exercise compared with before, and 24 hours after activity. The significant changes in testosterone and cortisol likely reflect the importance of volume, duration, and intensity of activity, and energy expenditure higher than the activity. More research is needed for better understanding of testosterone and cortisol in different time periods (for example, one hour, four hours, and 48 hours after exercise) to measure possible changes following acute combination exercise. 


\section{Acknowledgments}

We appreciate all the participants in this study and also Sports Laboratory of Faculty of Sport Sciences, Shahid Chamran University of Ahvaz.

\section{Ethical Approval}

The Institute for Advanced Studies of Shahid Chamran University Approved this study in 2014.

\section{Competing Interests}

Authors declare that they have no competing interests.

\section{References}

1. Mahdaviyan S, Behboodi L, Ezadi M. the effect of endurance training on the ratio of serum cortisol to dehydroepiandrosterone (DHEA) in inactive young women. International Journal of Basic Sciences \& Applied Research. 2015;4(1):38-43.

2. Agha-Alinejad H, Kohanpour MA, Sanavi S, Sojudi S, Behrouzi GR, Mirsepasi M. Effects of resistance training on serum cortisol and dehydroepiandrosterone levels in trained young women. Iran J Patholo. 2013;8(1):9-16.

3. Shakeri N, Nikbakht H, Azarbayjani M, Amirtash A. The effect of different types of exercise on the testosterone/ cortisol ratio in untrained young males. Ann Biol Res. 2012;3(3):1452-1460.

4. Beni MA, Akbari Z, Assarzadeh M, Azizbeigi K. The effect of selected aerobic training on serum immunoglobulin levels and testosterone and cortisol hormones in young men. Int J Sport Stud. 2013;3(9):956-962.

5. Hammes A, Andreassen TK, Spoelgen R, et al. Role of endocytosis in cellular uptake of sex steroids. Cell. 2005; 122(5):751-762. doi:10.1016/j.cell.2005.06.032.

6. Cardinale M, Soiza RL, Leiper JB, Gibson A, Primrose WR. Hormonal responses to a single session of wholebody vibration exercise in older individuals. Br J Sports Med. 2010;44(4):284-248. doi:10.1136/bjsm.2007.043232.

7. Izquierdo M, Häkkinen K, Ibanez J, et al. Effects of strength training on muscle power and serum hormones in middleaged and older men. J Appl Physiol. 2001;90(4):1497-1507.

8. Kraemer WJ, Loebel CC, Volek JS, et al. The effect of heavy resistance exercise on the circadian rhythm of salivary testosterone in men. Eur J Appl Physiol. 2001;84(1-2):1318. doi: $10.1007 / \mathrm{s} 004210000322$.

9. Dabbs JM, Dabbs MG. Heroes, rogues, and lovers: Testosterone and behavior. McGraw-Hill; 2000.

10. Deschenes MR, Kraemer WJ, Bush JA, et al. Biorhythmic influences on functional capacity of human muscle and physiological responses. Med Sci Sports Exerc. 1998;30(9):1399-1407. doi:10.1097/00005768-19980900000008 .

11. Buono MJ, Yeager JE, Hodgdon JA. Plasma adrenocorticotropin and cortisol responses to brief highintensity exercise in humans. J Appl Physiol. 1986;61(4): 1337-1379. doi:10.1249/00005768-198604001-00064.

12. Kraemer WJ, Ratamess NA. Hormonal responses and adaptations to resistance exercise and training. Sports Med. 2005;35(4):339-361.

13. Kraemer WJ, Rogol AD. The Endocrine System in Sports and Exercise. Wiley; 2005.

14. Miller JD. The effect of exercise order on testosterone and cortisol responses to lower and upper body resistance training exercises. Utah: The University of Utah; 2010.
15. Dudley GA, Djamil R. Incompatibility of enduranceand strength-training modes of exercise. J Appl Physiol. 1985;59(5):1446-1451.

16. Tarpenning KM, Hawkins SA, Marcell TJ, Wiswell RA. Endurance exercise and leg strength in older women. J Aging Phys Act.. 2006;14(1): 3.

17. Portegijs E, Kallinen M, Rantanen T, et al. Effects of resistance training on lower-extremity impairments in older people with hip fracture. Arch Phys Med Rehabil. 2008;89(9):1667-1674. doi:10.1016/j.apmr.2008.01.026.

18. Hoffman J. Physiological aspects of sport training and performance. Human Kinetics; 2014.

19. Sillanpää E, Laaksonen DE, Häkkinen A, et al. Body composition, fitness, and metabolic health during strength and endurance training and their combination in middleaged and older women. Eur J Appl Physiol. 2009;106(2): 285-296. doi:10.1007/s00421-009-1013-x.

20. Hawley JA. Molecular responses to strength and endurance training: Are they incompatible? This paper article is one of a selection of papers published in this Special Issue, entitled 14th International Biochemistry of Exercise ConferenceMuscles as Molecular and Metabolic Machines, and has undergone the Journal's usual peer review process. Appl Physiol Nutr Metab. 2009;34(3):355-361.

21. Brownlee KK, Moore AW, Hackney AC. Relationship between circulating cortisol and testosterone: influence of physical exercise. J Sports Sci Med. 2005;4(1):76.

22. Enea C, Boisseau N, Ottavy M, et al. Effects of menstrual cycle, oral contraception, and training on exercise-induced changes in circulating DHEA-sulphate and testosterone in young women. Eur J Appl Physiol. 2009;106(3):365-373.

23. Tremblay MS, Copeland JL, Van Helder W. Influence of exercise duration on post-exercise steroid hormone responses in trained males. Eur J Appl Physiol. 2005; 94(56): 505-13. doi:10.1007/s00421-005-1380-X

24. Fry A, Lohnes C. Acute testosterone and cortisol responses to high power resistance exercise. Hum Physiol. 2010; 36(4):457-461. doi:10.1134/S0362119710040110.

25. Linnamo V, Pakarinen A, Komi PV, Kraemer WJ, Häkkinen $\mathrm{K}$. Acute hormonal responses to submaximal and maximal heavy resistance and explosive exercises in men and women. J Strength Cond Res. 2005;19(3):566-71.

26. Schwanbeck SR. The Effects of Training with Free Weights or Machines on Muscle Mass, Strength, and Testosterone and Cortisol Levels. Saskatoon: University of Saskatchewan; 2008.

27. Hill E, Zack E, Battaglini C, Viru M, Viru A, Hackney A. Exercise and circulating cortisol levels: the intensity threshold effect. J Endocrinol Invest. 2008;31(7):587-591. doi:10.1007/BF03345606

28. Rosa G, Dantas E, Biehl C, de Castro e Silva H, Montano M, de Mello D. Leptin, Cortisol and Distinct Concurrent Training Sequences. Int J Sports Med. 2012;33(3):177. doi:10.1055/s-0031-1298002.

29. Cruz IS, Rosa G, Valle V, Mello DB, Fortes M, Dantas EH. Acute effects of concurrent training on serum leptin and cortisol in overweight young adults. Revista Brasileira de Medicina do Esporte. 2012;18(2): 81-86.

30. Cadore EL, Izquierdo M, dos Santos MG, et al. Hormonal responses to concurrent strength and endurance training with different exercise orders. J Strength Cond Res.2012; 26(12):3281-3288.

31. Brzycki M. Strength testing-predicting a one-rep max from reps-to-fatigue. Journal of Physical Education, 
Recreation \& Dance. 1993;64(1):88-90. doi:10.1080/07303 084.1993.10606684

32. Pollock ML, Gaesser GA, Butcher JD, et al. ACSM position stand: the recommended quantity and quality of exercise for developing and maintaining cardiorespiratory and muscular fitness, and flexibility in healthy adults. Med Sci Sports Exerc. 1998;30(6): 975-991.

33. Medicine ACoS. ACSM's guidelines for exercise testing and prescription: Lippincott Williams \& Wilkins; 2013.

34. Ainsworth BE, Haskell WL, Herrmann SD, et al. 2011 Compendium of Physical Activities: a second update of codes and MET values. Med Sci Sports Exerc. 2011;43(8): 1575-1581.

35. Uchida MC, Nosaka K, Ugrinowitsch C, et al. Effect of bench press exercise intensity on muscle soreness and inflammatory mediators. J Sports Sci. 2009;27(5): 499-507. doi:10.1080/02640410802632144.

36. Orsatti FL, Nahas EA, Maesta N, Nahas-Neto J, Burini RC. Plasma hormones, muscle mass and strength in resistancetrained postmenopausal women. Maturitas. 2008;59(4): 394-404. doi:10.1016/j.maturitas.2008.04.002

37. Kraemer RR, Chu H, Castracane VD. Leptin and exercise. Exp Biol Med (Maywood). 2002;227(9):701-708.

38. Goto K, Higashiyama M, Ishii N, Takamatsu K. Prior endurance exercise attenuates growth hormone response to subsequent resistance exercise. Eur J Appl Physiol. 2005; 94(3): 333-338. doi:10.1007/s00421-004-1296-x

39. Smilios I, Pilianidis T, Karamouzis M, Tokmakidis SP. Hormonal responses after various resistance exercise protocols. Med Sci Sports Exerc. 2003;35(4):644-54.

40. Kraemer WJ, Marchitelli L, Gordon SE, et al. Hormonal and growth factor responses to heavy resistance exercise protocols. J Appl Physiol. 1990;69(4):1442-50.

41. Häkkinen K, Pakarinen A, Newton R, Kraemer W. Acute hormone responses to heavy resistance lower and upper extremity exercise in young versus old men. Eur J Appl Physiol Occup Physiol. 1998;77(4):312-319.

42. Cadore EL, Lhullier FL, Alberton CL, et al. Salivary hormonal responses to different water-based exercise protocols in young and elderly men. The Journal of Strength \& Conditioning Research. 2009;23(9):2695-2701.

43. French DN, Kraemer WJ, Volek JS, et al. Anticipatory responses of catecholamines on muscle force production. J Appl Physiol. 2007;102(1):94-102. doi:10.1152/ japplphysiol.00586.2006.
44. Kraemer W, Gordon S, Fleck S, et al. Endogenous anabolic hormonal and growth factor responses to heavy resistance exercise in males and females. Int J Sports Med. 1991;12: 228-235.

45. Lu SS, Lau CP, Tung Y, et al. Lactate and the effects of exercise on testosterone secretion: evidence for the involvement of a cAMP-mediated mechanism. Med Sci Sports Exerc. 1997;29(8):1048-54. doi:10.1097/00005768199708000-00010.

46. McCall GE, Byrnes WC, Fleck SJ, Dickinson A, Kraemer WJ. Acute and chronic hormonal responses to resistance training designed to promote muscle hypertrophy. Canadian J Appl Physiol. 1999; 24(1):96-107.

47. Schwab R, Johnson GO, Housh TJ, Kinder JE, Weir J. Acute effects of different intensities of weight lifting on serum testosterone. Med Sci Sports Exerc. 1993;25(12):13811385. doi:10.1249/00005768-199312000-00011.

48. Rosa G, Cruz L, De Mello DB, De Sa Rego Fortes M, Dantas EH. Plasma levels of leptin in overweight adults undergoing concurrent training: original research article. Int Sport Med J. 2010;11(3):356-362.

49. Tremblay MS, Copeland JL, Van Helder W. Effect of training status and exercise mode on endogenous steroid hormones in men. J Appl Physiol. 2004;96(2):531-539. doi:10.1152/japplphysiol.00656.2003

50. Kraemer W, Noble B, Clark M, Culver B. Physiologic responses to heavy-resistance exercise with very short rest periods. Int J Sports Med. 1987; 8(4): 247-52.

51. Rezaee S, Hedayati M. Comparison of acute hormonal responses between resistance, endurance and enduranceresistance exercise in healthy young men. Physiol Pharmacol. 2011;14(4):445-457.

52. Alghadir AH, Gabr SA, Aly FA.The effect of foour weeks aerobic traininig on saliva cortisol and testosterone in young healthy persons. J Phys Ther Sci. 2015;27(7):20292033. doi:10.1589/jpts.27.2029

53. Mohamadi S, Khoshdel A, Naserkhani F, Mehdizadeh R. The effect of low - intensity resistance training with blood flow restriction on serum cortisol and testosterone levels in young men. Arch Mil Med. 2015;3(3):e28306. doi:10.5812/ jamm.28306.

54. Elahi A, Atashak S. The consumption effects of walnut oil extract supplement on inflammatory markers of IL-6 and cortisol hormone on active girls after an exhaustive exercise session. Int J Sport Stud. 2015;5(7):828-833. 